\title{
Fatores associados ao consumo alimentar na escola e ao sobrepeso/ obesidade de escolares de 7-10 anos de Santa Catarina, Brasil
}

\author{
Factors associated with food consumption in schools \\ and overweight/obesity in 7 to 10-year-old schoolchildren \\ in the state of Santa Catarina, Brazil
}

Camila Elizandra Rossi ${ }^{1}$

Larissa da Cunha Feio Costa ${ }^{1}$

Manuella de Souza Machado ${ }^{1}$

Dalton Francisco de Andrade ${ }^{2}$

Francisco de Assis Guedes de Vasconcelos ${ }^{1}$

${ }^{1}$ Programa de PósGraduação em Nutrição, Centro de Ciências da Saúde, Universidade Federal de Santa Catarina (UFSC). R. Delfino Conti s/n, Trindade. 88040-900 Florianópolis SC Brasil. camilarossi@uffs.edu.br

${ }^{2}$ Centro Tecnológico, Departamento de Informática e Estatística, UFSC. Florianópolis SC Brasil.

\begin{abstract}
The scope of this article is to investigate the association between the source of snacks consumed in schools, their nutritional value and overweight/obesity. It involved a cross-sectional study with 3,930 schoolchildren aged between 7 and10 in the state of Santa Catarina. Food consumption in the school was assessed with an illustrated 24-hour dietary recall and the source of snacks was investigated by structured interview. Soft drinks and artificial fruit juices, fried and processed snacks, ready-to-eat or sweetened snacks, delicacies and stuffed biscuits were classified as "low nutritional value foods," while fruit, natural fruit juices, vegetables, and vegetable soup were the "high nutritional value foods." Univariate and multiple logistic regression was conducted. Overweight/obesity was significantly associated with higher food consumption from the school canteen $(O R=1.34 ; C I=1.07-1.68)$ in private schools. Bringing food from home was significantly associated with increased consumption of low nutritional foods, both in public (OR = 1.56; $C I$ $=1.32-1.83)$ and private $(O R=2.64 ; C I=1.76$ 3.97) schools. The results highlight the need for tightening of norms to reduce the availability of low nutritional value food in canteens, and actions to promote nutritional education involving the school community.
\end{abstract}

Key words Obesity, School food, Food consumption, Schoolchildren
Resumo Oobjetivo deste artigo é investigar associação entre a proveniência de lanches consumidos na escola, seu valor nutricional e o sobrepeso/obesidade. Estudo transversal com 3.930 escolares de 7-10 anos de idade, de Santa Catarina. O consumo alimentar na escola foi avaliado com R-24 horas ilustrado, e a origem dos lanches por entrevista estruturada. Refrigerantes e sucos artificiais, frituras, salgadinhos industrializados, lanches prontos, doces, guloseimas e biscoitos recheados foram classificados como "lanches de baixo valor nutricional", enquanto frutas, sucos naturais, legumes, verduras e sopa de verduras foram os "lanches de alto valor nutricional". Realizou-se regressão logistica univariada e múltipla. O sobrepeso/obesidade se associou significativamente ao consumo de alimentos provenientes da cantina ( $O R=1,34$; IC $=1,07-1,68)$, na rede particular. Trazer alimentos de casa foi significativamente associado ao maior consumo de lanches de baixo valor nutricional, nas escolas públicas $(O R=1,56 ; I C=1,32-1,83) e$ nas particulares ( $O R=2,64 ; \mathrm{IC}=1,76-3,97)$. Os resultados apontam a necessidade de expansão de normativas que minimizem a disponibilidade de alimentos de baixo valor nutricional nas cantinas e que promovam educação nutricional envolvendo a comunidade escolar.

Palavras-chave Obesidade, Alimentação escolar, Consumo alimentar, Escolares 


\section{Introdução}

A obesidade é considerada um dos principais problemas contemporâneos de saúde pública no contexto mundial, acometendo todas as faixas etárias da população ${ }^{1,2}$. Especial atenção tem sido prestada à ocorrência da obesidade em crianças e adolescentes, uma vez que esta pode representar fator preditivo para obesidade em adultos ${ }^{3}$ ou ainda para doenças como dislipidemias, hipertensão e diabetes mellitus, anteriormente evidenciadas apenas em adultos ${ }^{4}$.

No Brasil, estudos realizados em diferentes locais do país revelam que, apesar das oscilações entre as regiões, a prevalência da obesidade em crianças em idade escolar é preocupante no país inteiro $^{5-8}$. Dados da última Pesquisa de Orçamentos Familiares (POF), coletados entre os anos de 2008 e 2009 em amostra nacionalmente representativa, mostram que a prevalência de obesidade atingia $15 \%$ das crianças brasileiras aos 7 anos de idade. E, se somadas as prevalências de sobrepeso e obesidade nestas crianças, o percentual chegava a 49\%, muito acima dos níveis esperados em saúde pública. Mesmo aos 10-11 anos de idade, quando as prevalências destes agravos mostravam redução, ainda assim o percentual se revelava preocupante, totalizando $35 \%$ destes adolescentes 9 . Na capital do estado de Santa Catarina, estudo com 1.223 escolares de 7 a 10 anos constatou prevalência de sobrepeso/obesidade em $32,7 \%$ das meninas e $36,2 \%$ em meninos ${ }^{10}$.

Os determinantes etiológicos da obesidade estão associados a uma combinação de fatores biológicos e ambientais, incluindo hábitos alimentares, propensão genética, estilo de vida, condição socioeconômica e fatores psicológicos ${ }^{5,8,10,11}$. Dentre os fatores relativos aos hábitos alimentares, destaca-se o consumo de alimentos de baixo valor nutricional e alta densidade energética ${ }^{12}$.

Com o início da vida escolar, a criança fica sujeita às influências nos padrões alimentares, sendo esta fase típica para a formação de hábitos e comportamentos que podem perdurar durante a vida adulta ${ }^{13}$. Dentro desta perspectiva, são desenvolvidas estratégias com o intuito de aproveitar a potencialidade da escola como espaço de promoção da saúde por meio da formação de hábitos alimentares saudáveis ${ }^{14-17}$. Estudos sobre o consumo alimentar no ambiente escolar revelam o alto consumo de alimentos de baixo valor nutricional e alta densidade energética, sejam estes alimentos adquiridos nas cantinas escolares, levados de casa para consumo na escola ${ }^{18,19}$ ou ainda fornecidos por programas públicos de alimentação escolar ${ }^{20}$. Além disso, há indícios da associação positiva entre obesidade e algumas características do ambiente alimentar nas escolas, tais como a oferta de doces e frituras ${ }^{21}$ e a disponibilidade de máquinas ou pontos de venda de alimentos e bebidas ${ }^{22,23}$.

Como parte da Política Nacional de Promoção da Saúde, o Ministério da Saúde do Brasil desenvolveu um conjunto de estratégias de promoção da alimentação saudável nas escolas, a fim de propiciar a adesão da comunidade escolar a hábitos alimentares saudáveis e atitudes de cuidado e promoção da saúde, chamado de "Dez Passos para a Promoção da Alimentação Saudável nas Escolas" ${ }^{24}$. Entretanto, são escassos estudos que avaliaram os lanches consumidos por escolares com base nessas orientações ou mesmo de estudos nacionais que identifiquem as diferenças entre os lanches trazidos de casa e aqueles adquiridos nas cantinas das escolas e suas influências no desenvolvimento do sobrepeso ou obesidade nas crianças.

Dessa forma, o presente artigo teve por objetivo investigar a associação entre a proveniência dos lanches consumidos na escola, seu valor nutricional e o sobrepeso/obesidade em escolares de 7 a 10 anos do ensino fundamental de Santa Catarina.

\section{Método}

Trata-se de um estudo transversal, com escolares regularmente matriculados entre o $2^{\circ}$ e o $5^{\circ}$ anos do Ensino Fundamental de escolas públicas e particulares, urbanas e rurais, situadas nos $8 \mathrm{mu}-$ nicípios pólos (caracterizados pelo maior número de escolas e escolares) do estado de Santa Catarina: Florianópolis, Joinville, Blumenau, Chapecó, Criciúma, Jaraguá do Sul, Lages e Joaçaba. Os dados foram coletados de junho de 2007 a abril de 2008. O desenho e desenvolvimento metodológico da investigação foram descritos com maiores detalhes em publicações anteriores ${ }^{25-27}$. Uma síntese de tais procedimentos será descrita na sequência.

\section{Amostragem}

O plano amostral comportou duas unidades de análise de interesse: a escola e o escolar. A seleção dos escolares foi realizada por conglomerados em três estágios, conforme segue:

Estágio 1 - primeiramente foram selecionadas as escolas participantes. Para compor o 
universo de escolas de Ensino Fundamental do Estado de Santa Catarina, as mesmas foram distribuídas em três regiões: oeste (Oeste e Meio-oeste), centro (Norte, Serrana e Alto Vale) e litoral (grande Florianópolis, litoral norte e sul). Na sequência, decidiu-se que, nestas regiões, as escolas deveriam estar situadas em oito municípios pólo, sendo estes, Blumenau, Chapecó, Criciúma, Jaraguá do Sul, Joaçaba, Joinville, Florianópolis e Lages. Tais municípios foram escolhidos intencionalmente com base no seguinte critério: ter o maior número de escolas e matrículas no ensino fundamental de $1^{\text {a }}$ a $5^{\text {a }}$ série. De acordo com dados do Censo Educacional do Ministério da Educação, em 2006 havia 4.007 escolas de Ensino Fundamental no estado, sendo uma federal, 3.661 escolas públicas (municipais e estaduais) e 345 particulares (http://www.inep.gov. br/censo/basica/dataescolabrasil/). Para o cálculo da amostra, foi excluída a escola federal (por representar apenas $0,03 \%$ das escolas) e aquelas que declararam não ter escolares nas séries iniciais do Ensino Fundamental. Para a definição final das escolas a serem investigadas, foram introduzidos outros critérios, como a dependência administrativa da escola (pública e particular) e o número de escolares matriculados (no mínimo 50 alunos). Dessa forma, o universo de estudo foi composto por 140.878 escolares matriculados em 569 escolas públicas e particulares dos oito municípios selecionados anteriormente. $\mathrm{O}$ número de escolas a ser investigado foi calculado de modo a garantir um erro amostral de no máximo 6 pontos percentuais, para mais ou para menos, para cada um dos seis estratos de interesse. Assim, a amostra final foi composta por 344 escolas (269 públicas e 75 particulares);

Estágio 2: seleção de uma turma em cada escola, por meio de sorteio aleatório simples;

Estágio 3: seleção de todos os alunos da turma amostrada.

A amostra final foi de 3.930 escolares. Foram excluídos aqueles que apresentavam inconsistência ou ausência de algum dado, idade acima de 10 anos, e cujos inquéritos alimentares de 24 horas foram aplicados nas segundas-feiras $(n=1.034$; $20,8 \%$ ), pois o objetivo deste estudo foi avaliar o consumo alimentar realizado na escola.

\section{Coleta de dados}

Os instrumentos foram avaliados e adequados por meio da realização de um teste piloto. A equipe responsável pela coleta dos dados foi composta por dez nutricionistas devidamente capacitados, conforme já descrito em estudos anteriores $^{25-27}$.

\section{Perfil antropométrico dos pais}

Conforme metodologia já validada ${ }^{28}$, optouse por utilizar informações autorreferidas por meio de um questionário socioeconômico composto por, dentre outros tópicos, questões acerca da idade, peso e estatura dos pais dos escolares. Com base na avaliação nutricional dos pais dos alunos, os escolares foram classificados como: filhos de pai e mãe não obesos, filhos de pai obeso e mãe não obesa, filhos de mãe obesa e pai não obeso, ou filhos de pai e mãe obesos. Os pais e mães dos escolares foram considerados não obesos sempre que apresentaram índice de massa corporal (IMC) inferior a $25,0 \mathrm{~kg} / \mathrm{m}^{2}$, e obesos quando apresentaram IMC igual ou maior que $25,0 \mathrm{~kg} / \mathrm{m}^{2}$, incluindo, desta forma, os pais e mães com sobrepeso dentro do grupo de obesos.

\section{Perfil antropométrico dos escolares}

A coleta das medidas antropométricas foi realizada na escola em horário de aula. Os escolares mantiveram-se descalços e usando o mínimo de roupa possível durante os procedimentos para obtenção das medidas antropométricas. A medida de massa corporal foi obtida por meio de balança eletrônica Marte ${ }^{\varpi}$, modelo PP180, com capacidade de $180 \mathrm{~kg}$ e precisão de 50 gramas. A medida da estatura foi obtida por meio da utilização de estadiômetro, marca Alturexata ${ }^{\varpi}$, com precisão de $1 \mathrm{~mm}$. Ambos os procedimentos seguiram as recomendações da Organização Mundial da Saúde - OMS (1995) 29.

Os escolares foram agrupados de acordo com o IMC/idade e sexo, a partir dos critérios adotados pela International Obesity Task Force (IOTF), considerando neste estudo as seguintes categorias: sem sobrepeso/obesidade (IMC/idade e sexo abaixo dos valores estabelecidos por Cole et al..$^{30}$ para diagnóstico de sobrepeso) e com sobrepeso/ obesidade (IMC/idade e sexo igual ou acima dos valores estabelecidos por Cole et al. ${ }^{30}$ para diagnóstico de sobrepeso e obesidade).

\section{Consumo alimentar na escola}

Para análise do consumo alimentar na escola foi utilizado o "Questionário Alimentar do dia Anterior” (QUADA). Trata-se de um Recordatório de 24 horas ilustrado, estruturado e validado para inquéritos alimentares direcionados a crian- 
ças em idade escolar ${ }^{31}$. O QUADA foi aplicado em sala de aula na forma de um exercício orientado, seguindo protocolo padronizado. Inicialmente foi realizada a explicação de cada grupo alimentar contido no instrumento, utilizando, como apoio, banners contendo as mesmas figuras dos questionários aplicados aos alunos. Em seguida os escolares eram orientados a circularem os alimentos consumidos em cada uma das refeições realizadas no dia anterior.

O questionário, subdividido em 6 refeições diárias ordenadas cronologicamente (café da manhã, lanche da manhã, almoço, lanche da tarde, jantar e ceia), é composto por representações gráficas em cada refeição, referentes a 17 grupos alimentares: bolachas salgadas sem recheio e pães; achocolatados com leite; lácteos (café com leite, leite, iogurte e queijo); arroz; refrigerantes e sucos artificiais; doces e guloseimas, bolos com cobertura e recheio e biscoitos recheados; salgadinhos industrializados de pacote; frituras (batata frita); lanches prontos (pizza, hambúrguer, fast foods); frutas; feijão e outras leguminosas; macarrão; peixe e frutos do mar; carne de gado e frango; suco natural; legumes; verduras e sopa de verduras ${ }^{31}$.

Foram consideradas refeições realizadas na escola, o lanche da manhã (para as crianças que estudam no período matutino), o lanche da tarde (para as crianças do vespertino) e ambos os lanches para crianças que estudam em escola integral.

Os grupos de alimentos foram reunidos conforme as diretrizes dos "Dez Passos para a Promoção da Alimentação Saudável nas Escolas" (a partir daqui denominado "Dez Passos"). Tais diretrizes definem os alimentos a terem o consumo restrito ou promovido nas escolas ${ }^{24}$. Com base nestes preceitos, os escolares foram agrupados quanto ao consumo de alimentos restritos pelos Dez Passos para consumo na escola (refrigerantes e sucos artificiais, frituras, salgadinhos industrializados, lanches prontos ou doces/guloseimas/ biscoitos recheados) que foram denominados "lanches de baixo valor nutricional" e alimentos promovidos pelos Dez Passos para consumo na escola (frutas, sucos naturais; legumes; verduras e sopa de verduras) na escola, que foram denominados "lanches de alto valor nutricional". Caso os escolares tenham registrado o consumo de pelo menos um dos itens destes grupos no dia anterior, por meio do QUADA, considerou-se que o escolar consumiu o alimento.

\section{Procedência dos alimentos consumidos na escola}

Informações sobre a adesão dos estudantes de escolas públicas ao Programa Nacional de Alimentação Escolar (PNAE) (http://www.fnde.gov. br/programas/alimentacao-escolar/alimentacao -escolar-apresentacao) - sim/não, ao hábito de aquisição de alimentos na cantina ( $\operatorname{sim} /$ não) e à prática de levar alimentos de casa para consumo na escola ( $\operatorname{sim} /$ não), bem como sobre a frequência semanal de consumo, foram coletadas por meio de aplicação de questionário estruturado, aplicado no mesmo dia da avaliação antropométrica. Os alunos responderam o questionário em sala de aula, sob orientação dos pesquisadores.

\section{Análise dos dados}

Os dados foram digitados no programa EpiData versão 3.1 por 4 digitadores devidamente treinados, utilizando-se checagem automática de consistência e amplitude. Após a digitação, foi realizada a conferência do banco na íntegra.

Para processamento e análise dos dados foi utilizado o programa Stata versão 14.0. Realizou-se inicialmente análise descritiva dos dados relativos ao consumo dos grupos alimentares na escola (alimentos restritos e promotores e procedência dos alimentos - casa, cantina ou PNAE), aplicando-se o teste de qui-quadrado de heterogeneidade de Pearson para verificar a diferença entre as prevalências das variáveis relativas aos escolares da rede pública e da rede particular.

Para verificar a associação entre os desfechos consumo de lanches restritos na escola, consumo de lanches promovidos na escola e sobrepeso/ obesidade com as variáveis explicativas, aplicaram-se Modelos de Regressão Logística Univariada e Múltipla do tipo forward. Neste modelo, todas as variáveis que apresentaram valor de $p$ $<0,25$ na regressão logística univariada foram adicionadas ao modelo múltiplo. Neste último, foi considerado estatisticamente significante a relação de variáveis com $\mathrm{p}<0,05$. Dessa forma, foram estimadas as razões de chances (RC) com intervalos de confiança de 95\%. Cabe ressaltar que o Modelo de Regressão Logística foi aplicado considerando o plano amostral, mediante utilização do recurso "svy". Tal procedimento possibilitou realizar a análise estatística considerando a real probabilidade de cada escolar ter sido selecionado para o estudo, visto que a primeira unidade de interesse selecionada foram as escolas, seguido das turmas e, por fim, os escolares. 


\section{Aspectos éticos da pesquisa}

O presente estudo teve aprovação do Comitê de Ética em Pesquisa com Seres Humanos da Universidade Federal de Santa Catarina/CCS. A mensuração dos dados antropométricos e a aplicação do inquérito de consumo alimentar com os escolares foram realizadas somente mediante devolução do "Termo de Consentimento Livre e Esclarecido para os pais dos alunos selecionados" assinado pelos pais ou responsáveis.

\section{Resultados}

Neste estudo foram analisados dados de 3.930 escolares de 7 a 10 anos (taxa de resposta de $87,3 \%)$, matriculados entre o $2^{\circ}$ e $5^{\circ}$ ano do ensino fundamental.

Considerando o tamanho de amostra ( $\mathrm{n}=$ 3.930), as prevalências das distintas variáveis de exposição e dos desfechos, assim como o ajuste para fatores de confusão, o presente estudo teve poder de $80 \%$ para detectar como estatisticamente significativa $(\mathrm{p}<0,05)$ razão de odds $(\mathrm{OR})$ entre 1,13 a 1,20 e 0,87 a $0,80^{32}$

A amostra avaliada constitui-se principalmente por escolares do sexo feminino, consumidores de alimentos do PNAE na rede pública, e filhos de pai obeso, especialmente entre escolares da rede particular de ensino. O consumo de lanches de baixo valor nutricional (refrigerantes e sucos artificiais, frituras, salgadinhos industrializados, lanches prontos ou doces/guloseimas/ biscoitos recheados) foi prevalente em 41,0\% dos escolares e, na rede pública, foi significativamente superior. Nesta rede de ensino, apesar da existência do PNAE, mais da metade dos escolares relataram trazer alimentos de casa e mais de $1 / 3$ deles relatou adquirir alimentos de cantinas. Os escolares da rede particular consumiram lanches de alto valor nutricional (frutas, sucos naturais; legumes; verduras e sopa de verduras) com frequência significativamente maior que os escolares da rede pública (Tabela 1).

Apesar de as meninas terem apresentado frequência maior de consumo de lanches de baixo valor nutricional quando comparadas aos meninos em ambas as redes de ensino, as diferenças entre os sexos não foram significativas. A frequência e a chance de consumir lanches de baixo valor nutricional na escola foram significativamente maiores entre aqueles que trouxeram alimentos de casa, tanto na rede pública quanto na rede particular. Na rede pública de ensino, po- rém, a chance de consumo dos lanches de baixo valor nutricional é 50\% menor entre os escolares que relataram ter consumido lanches de alto valor nutricional no dia anterior. Ou seja, entre estudantes que escolheram ingerir itens saudáveis, seja qual for a sua proveniência, houve menor chance de consumo de itens de baixo valor nutricional. Nas escolas particulares, a chance de consumo de lanches de baixo valor nutricional foi significativamente menor entre filhos de mãe obesa (Tabela 2).

Em ambas as redes de ensino, o perfil antropométrico dos pais se associou inversa e significativamente ao consumo de alimentos promovidos pelos "Dez passos para a alimentação saudável na escola" (lanches de alto valor nutricional). $\mathrm{Ou}$ seja, houve menor chance de consumo de lanches de alto valor nutricional no ambiente escolar entre os estudantes de escolas públicas que tinham pelo menos um genitor com obesidade e também menor chance de consumo destes lanches nas escolas particulares quando ambos os pais tinham obesidade. Os escolares de oito anos apresentaram chance significativamente menor de consumirem lanches de alto valor nutricional nas escolas públicas e maior chance de consumo destes alimentos nas escolas particulares (Tabela 3 ).

Nas escolas públicas, o estado nutricional dos pais encontra-se diretamente associado ao estado nutricional dos escolares, de modo que filhos de pai e mãe obesos possuem chances significativamente maiores de serem obesos quando comparados aos demais, e escolares da rede particular com o pai obeso é que possuem maior chance de apresentar sobrepeso/obesidade. Além disso, na rede particular, o fato de os escolares consumirem alimentos nas cantinas aumentou as chances de sobrepeso/obesidade (Tabela 4).

\section{Discussão}

De acordo com os resultados apresentados, o consumo de lanches de baixo valor nutricional no ambiente escolar é preocupante, especialmente entre os escolares da rede pública, os quais são beneficiários do PNAE, programa que segue premissas legais de oferta de alimentação nutricionalmente adequada ${ }^{33}$

Nas duas edições da Pesquisa Nacional de Saúde do Escolar (PENSE), realizada com escolares do $9^{\circ}$ ano do ensino fundamental no Brasil, nos anos de 2012 e $2015^{34,35}$, este problema de elevado consumo de alimentos marcadores de alimentação não saudável também foi identifica- 
Tabela 1. Caracterização dos escolares investigados, por rede administrativa da escola. Florianópolis, 2007.

\begin{tabular}{|c|c|c|c|c|}
\hline \multirow[t]{2}{*}{ Variáveis } & Total & $\begin{array}{l}\text { Escolas } \\
\text { Públicas }\end{array}$ & $\begin{array}{c}\text { Escolas } \\
\text { Particulares }\end{array}$ & \multirow[t]{2}{*}{ p-valor ${ }^{\mathrm{a}}$} \\
\hline & $\%(n=3.930)$ & $\%(n=3.049)$ & $\%(n=881)$ & \\
\hline \multicolumn{5}{|l|}{ Sexo } \\
\hline Masculino & $48,0(1887)$ & $47,7(1469)$ & $48,1(418)$ & \multirow[t]{2}{*}{0,860} \\
\hline Feminino & $52,0(2043)$ & $52,3(1580)$ & $51,9(463)$ & \\
\hline \multicolumn{5}{|l|}{ Idade } \\
\hline 7 & $23,0(869)$ & $21,5(596)$ & $33,3(273)$ & \multirow{4}{*}{0,049} \\
\hline 8 & $29,7(1133)$ & $29,5(867)$ & $31,1(266)$ & \\
\hline 9 & $29,4(1141)$ & $30,2(940)$ & $23,8(201)$ & \\
\hline 10 & $17,9(787)$ & $18,8(646)$ & $11,8(141)$ & \\
\hline \multicolumn{5}{|c|}{ Locais de procedência dos lanches consumidos na escola } \\
\hline Casa & $53,9(2063)$ & $52,0(1480)$ & $67,4(583)$ & $<0,001$ \\
\hline Cantina & $35,0(1266)$ & $32,4(827)$ & $52,2(439)$ & 0,003 \\
\hline PNAE & $71,3(2601)$ & $81,2(2601)^{*}$ & - & - \\
\hline \multicolumn{5}{|l|}{ Consumo de lanches na escola } \\
\hline De baixo valor nutricional ${ }^{\star \star}$ & $41,0(1672)$ & $74,6(1199)$ & $57,0(473)$ & 0,009 \\
\hline De alto valor nutricional ${ }^{\star * \star}$ & $26,4(1046)$ & $25,4(743)$ & $33,3(303)$ & 0,009 \\
\hline \multicolumn{5}{|l|}{ Perfil antropométrico dos escolares } \\
\hline Sem sobrepeso/obesidade & $79,1(3077)$ & $79,6(2412)$ & $75,9(665)$ & \multirow[t]{2}{*}{0,035} \\
\hline Com sobrepeso/obesidade & $20,9(853)$ & $20,4(637)$ & $24,1(216)$ & \\
\hline \multicolumn{5}{|l|}{ Perfil antropométrico dos Pais } \\
\hline Mãe e Pai não obesos & 30,7 (949) & $30,4(698)$ & $32,5(251)$ & \multirow[t]{5}{*}{$<0,001$} \\
\hline Pai Obeso e Mãe não obesa & $32,0(1044)$ & $29,7(706)$ & $46,7(338)$ & \\
\hline Mãe Obesa e Pai não obeso & $15,1(432)$ & $16,8(399)$ & $4,4(33)$ & \\
\hline Mãe e Pai obesos & $22,2(707)$ & $23,1(574)$ & $16,4(133)$ & \\
\hline Total & $100,0(3.132)$ & $100,0(2.377)$ & $100,0(755)$ & \\
\hline
\end{tabular}

do entre os escolares. Em 2012, 41,3\% dos escolares referiram consumir lanches de baixo valor nutricional (doces, balas, chocolates, chicletes, bombons ou pirulitos) e 33,2\% referiram ingerir refrigerantes, em cinco dias ou mais na semana. Em 2015, 13,7\% dos escolares refeririam consumir salgados fritos, 41,6\% guloseimas, 26,7\% refrigerantes e $31,3 \%$ ultraprocessados salgados em frequência igual ou superior a cinco dias na semana. Quanto ao consumo de alimentos marcadores de alimentação saudável (alto valor nutricional), em 2012, 69,9\% dos escolares consumiram feijão; 43,4\%, hortaliças e 30,2\%, frutas frescas, enquanto que em 2015, esses percentuais atingiram $60,7 \%$ para feijão, $37,7 \%$ para legumes e $32,7 \%$ para frutas frescas.

Entretanto, no presente estudo não somente o consumo em si de lanches de baixo valor nutricional causa preocupação, mas o fato de, em ambas as redes de ensino, os alimentos trazidos de casa estarem associados a este consumo ali- mentar preocupante, indicando que as escolhas de alimentos entre os familiares não está seguindo as premissas da alimentação saudável. Estudo anterior realizado com escolares catarinenses, de duas escolas de Florianópolis, indica a bolacha recheada como o alimento mais trazido de casa para consumo nas escolas públicas e particula$\mathrm{res}^{36}$. Outro estudo realizado no Brasil ${ }^{37}$ e pesquisas realizadas nos Estados Unidos da America ${ }^{19}$, na Austrália ${ }^{17}$ e na Arábia Saudita ${ }^{38}$ também relatam o alto consumo de alimentos de alta densidade energética nas escolas. De acordo com as estratégias propostas pelos "Dez Passos para Promoção da Alimentação Saudável na Escola"24 e com as diretrizes da Portaria $n^{\circ}$ 1.010/MS/MEC de $2006^{39}$, é necessário que a escola envolva toda comunidade escolar (inclusive os pais dos alunos) nas atividades de promoção da saúde.

Santa Catarina possui uma legislação que veta a comercialização de alimentos como balas, pirulitos, refrigerantes, sucos artificiais, frituras 


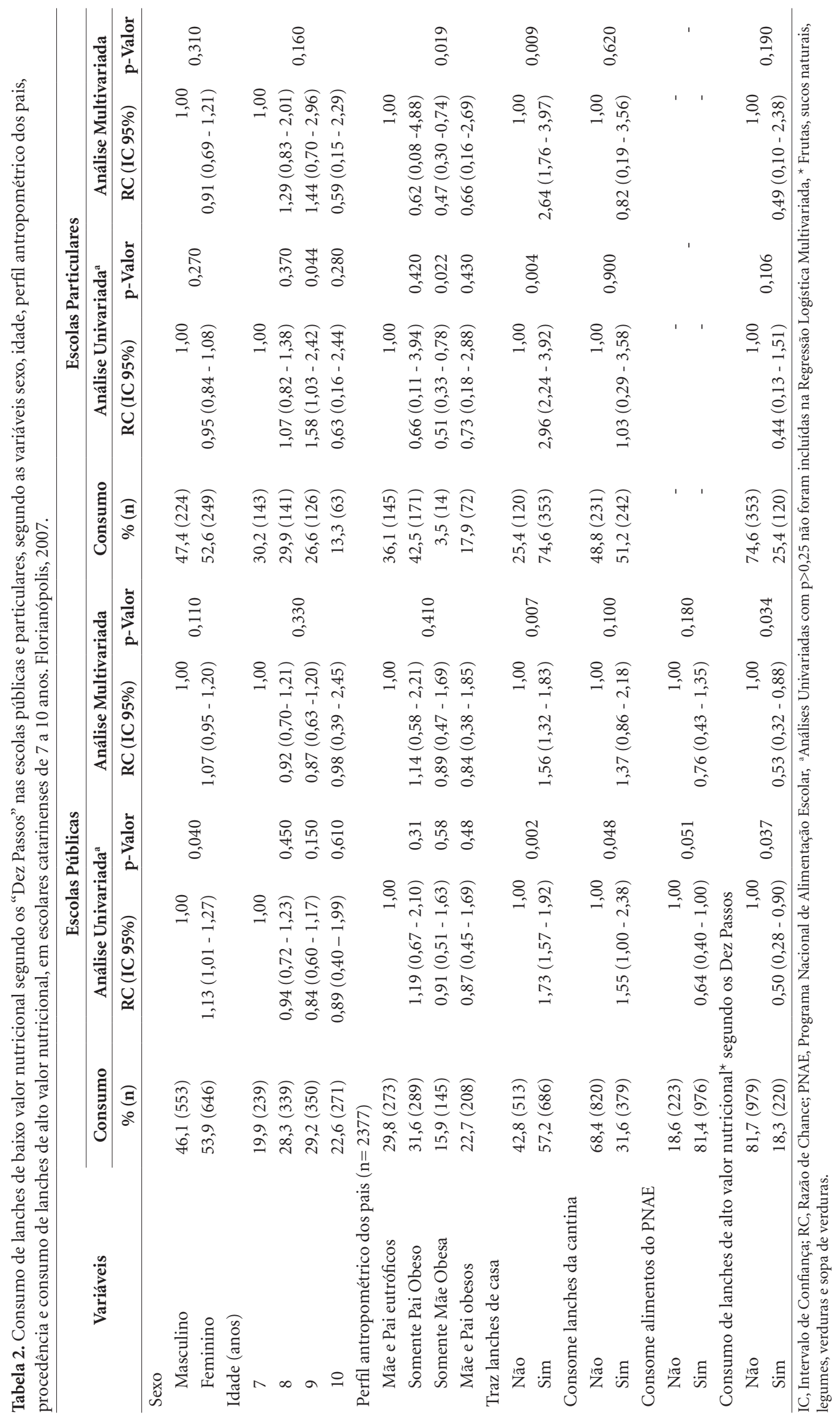




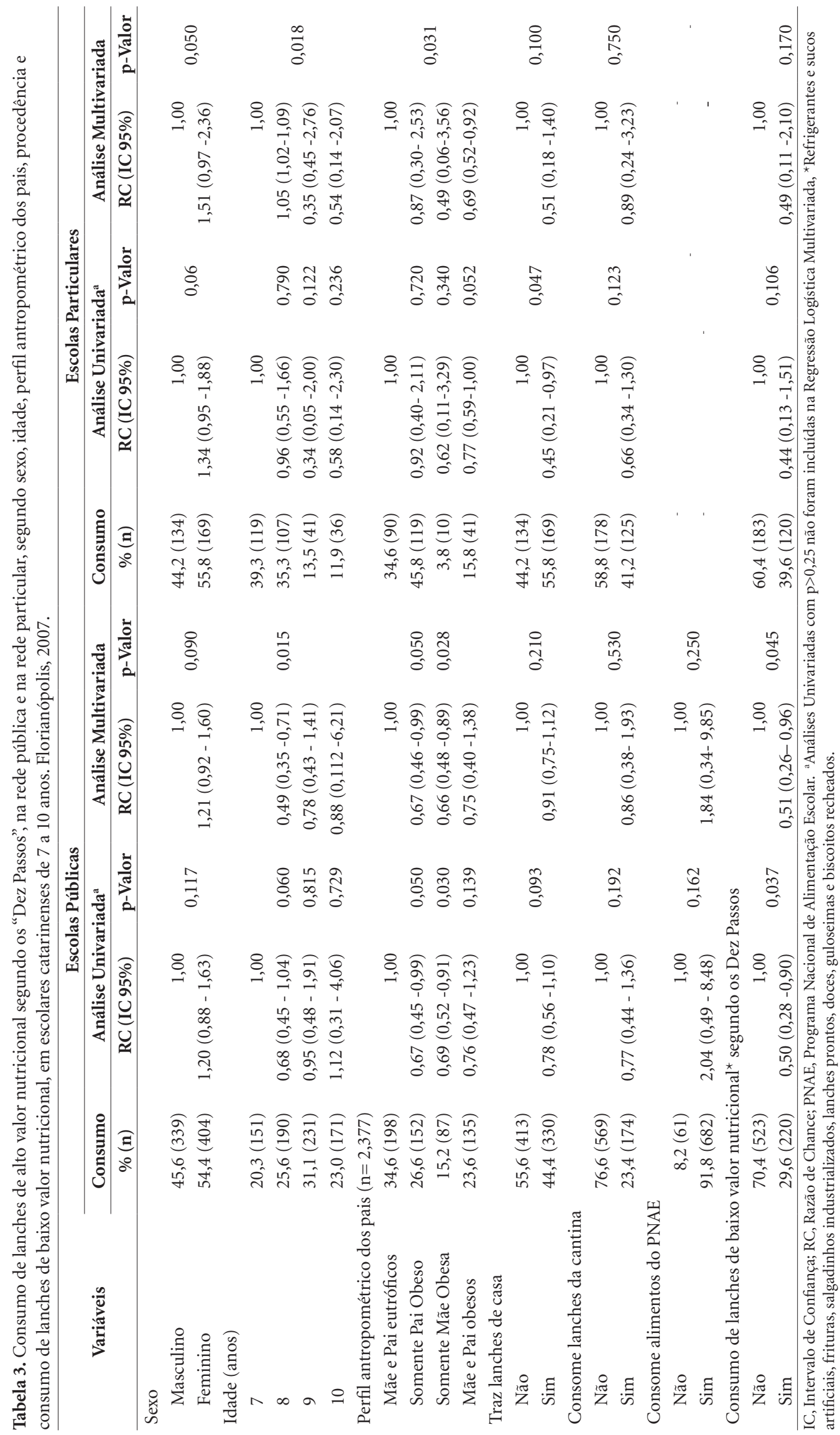




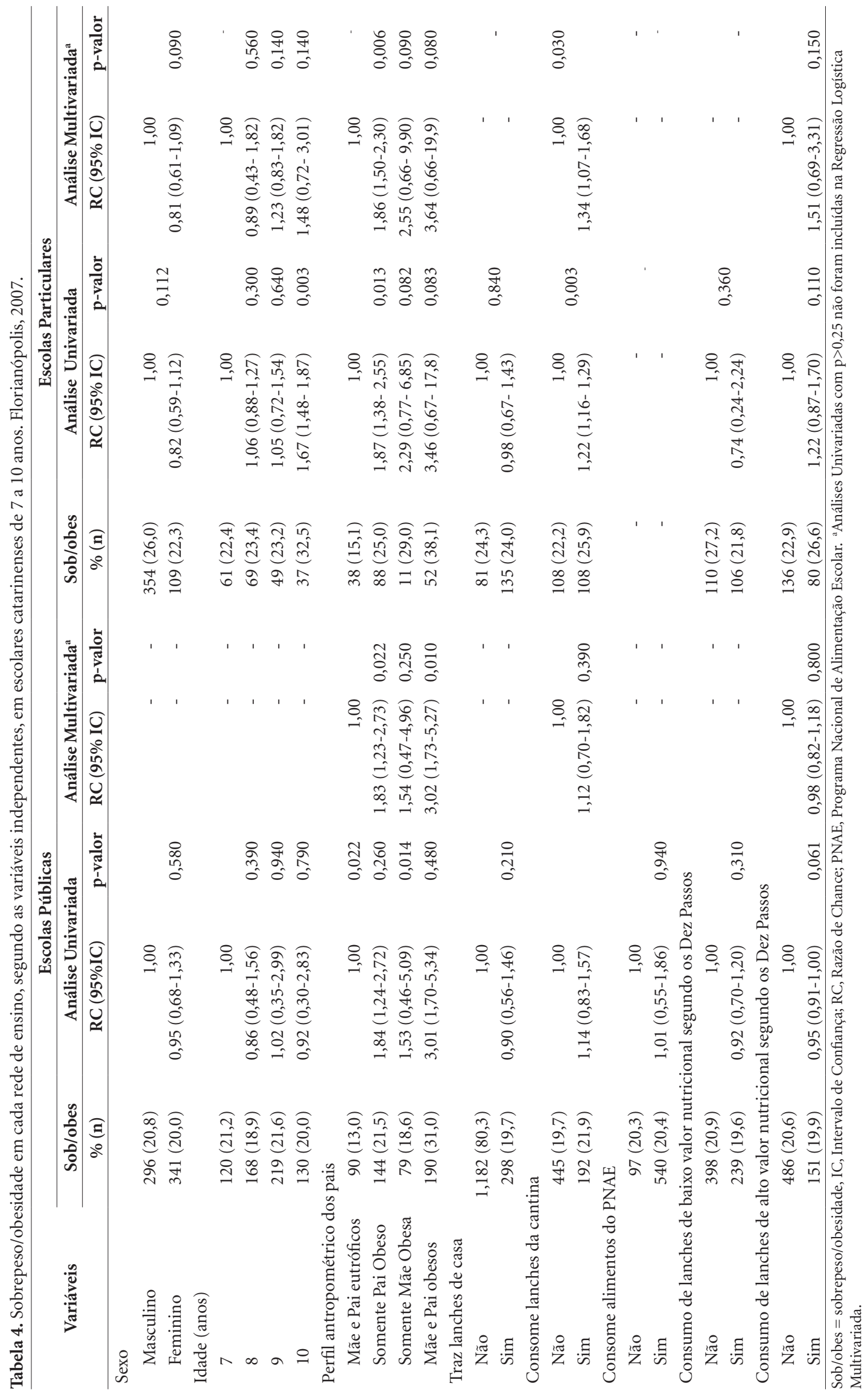


e salgadinhos industrializados e torna obrigatório o oferecimento de pelo menos duas opções de frutas diárias em quaisquer estabelecimentos de comercialização de alimentos das escolas catarinenses ${ }^{40}$. Avaliação do cumprimento desta lei nas cantinas das escolas catarinenses revelou que $68,2 \%$ das cantinas realmente não comercializam nenhum dos alimentos de baixo valor nutricional e $16,8 \%$ ofereciam frutas diariamente ${ }^{22}$. Tal fato pode ser sugerido como justificativa para a associação não significativa entre consumir alimentos das cantinas com a ingestão de alimentos de baixo valor nutricional nas escolas públicas, sugerindo que a lei das cantinas pode estar resultando em menor frequência de consumo de alimentos nutricionalmente inadequados (baixo valor nutricional). Por outro lado, o fato de poucas das cantinas catarinenses oferecerem as frutas também pode explicar a associação não significativa encontrada entre ingerir alimentos adquiridos nestes estabelecimentos e o consumo de lanches de alto valor nutricional, promovidos pelos Dez Passos para uma Alimentação Saudável.

A Lei $n^{\circ} 11.947$ de 16 de junho de $2009^{33} \mathrm{e}$ a Resolução nº 26/2013 do Fundo Nacional de Desenvolvimento da Educação (FNDE) ${ }^{41}$ dispõem sobre o atendimento à alimentação escolar e determinam, dentre outras deliberações, que a alimentação escolar deve ser saudável e adequada, compreendendo o uso de alimentos variados que respeitem a cultura e os hábitos alimentares saudáveis, em conformidade com faixa etária e estado de saúde do escolar. A mesma normatização reafirma que os cardápios do PNAE devam estar sob a responsabilidade técnica de um nutricionista, tal como já definia a Resolução no 32 , de $10 / 8 / 2006^{42}$, vigente na época da coleta dos dados deste estudo. O fato de os cardápios do PNAE serem elaborados por nutricionistas, conforme os preceitos de uma alimentação saudável, deveria colaborar para uma associação significativa com o consumo de alimentos protetores, resultado que não foi encontrado. Vale destacar que o consumo de alimentos da escola foi avaliado por meio de apenas um Recordatório de 24 horas, o que pode ter colaborado para não se encontrar associação entre as variáveis. Uma pesquisa com 2.314 escolares dos Estados Unidos da América aponta que consumidores do School Lunch Program (SLP) e do School Breakfast Program (SBP) apresentam melhor perfil dietético na escola. De acordo com os autores, escolares usuários do SLP consomem mais leite, frutas, sucos naturais e menos sobremesas e snacks ( $\mathrm{p}<0,01$ ), enquanto usuários do SBP ingerem mais leite, frutas e sucos naturais e menos bebidas (que não leite ou suco natural $)(\mathrm{p}<0,01)^{43}$. Uma análise de três dias alimentares das escolas catarinenses, portanto, é sugerida para estudos futuros, na expectativa de se encontrarem resultados similares aos supracitados.

Corroborando com diversos estudos internacionais ${ }^{44-47}$, os resultados encontrados não detectaram associação do estado nutricional dos escolares com o consumo de alimentos de casa na escola ou do PNAE. Entretanto, verificou-se associação positiva do consumo de alimentos nas cantinas particulares com a obesidade, provavelmente em função destes estabelecimentos ainda oferecerem alimentos inadequados, bem como devido à maior disposição deste tipo de estabelecimento nas escolas particulares ${ }^{48}$. Gabriel et al. ${ }^{25}$ já indicavam a necessidade de maior fiscalização da legislação que regulamenta a comercialização de alimentos nas cantinas escolares ${ }^{22}$. Da mesma forma que em estudo realizado 1.223 escolares de 7 a 10 anos de Florianópolis, a presente pesquisa também verificou associação positiva entre a obesidade e o estado nutricional dos pais. Os autores da referida pesquisa indicam a necessidade de intervenções também no ambiente familiar'.

Dentre as limitações do presente estudo, ressalta-se que apesar de o instrumento utilizado na análise do consumo alimentar ser específico para crianças em fase escolar, os dados representam apenas o consumo realizado em um único dia e o instrumento utilizado não permite a identificação das quantidades exatas dos alimentos ingeridos, apenas o tipo ou qualidade nutricional dos mesmos e o número de refeições em que foram ingeridos durante um dia ${ }^{28}$. Além disso, ainda que o consumo alimentar na escola seja importante na vida de crianças e adolescentes, a maior parte da ingestão calórica deve ser realizada fora da escola. Finalmente, o caráter multifatorial da obesidade envolve diversos fatores (a exemplo, de variáveis relacionadas ao ambiente geográfico e familiar, bem como à atividade física e outros fatores do estilo de vida) não considerados neste estudo.

Os resultados explicitam não somente a necessidade de expansão das medidas legislativas com vistas a minimizar a disponibilidade de alimentos de risco nas cantinas escolares no âmbito nacional, como enfatiza a necessidade de fiscalização eficaz destes estabelecimentos.

Tais medidas devem ser acompanhadas do aprimoramento do Programa Nacional de Alimentação Escolar, no sentido de promover atividades de educação nutricional que envolvam a 
comunidade escolar, inclusive pais de escolares e cantineiros. Estratégia esta que pode auxiliar os pais na seleção de alimentos mais adequados a serem levados de casa para consumo nas escolas pelos escolares, bem como na maior disponibilidade/consumo de alimentos saudáveis nas cantinas. Dentro desta perspectiva, enfatiza-se a relevância do profissional nutricionista como agente de transformação e conscientização de alunos e da comunidade escolar, promovendo a saúde por meio da educação alimentar e nutricional.

\section{Referências}

1. World Health Organization (WHO). Obesity: preventing and managing the global epidemic. Geneva: WHO; 2000. (Technical Report Series, n. 894).

2. World Health Organization (WHO). Obesity and overweight. WHO Media Centre, 2016. [acessado 2016 Out 03]. Disponível em: http://www.who.int/mediacentre/ factsheets/fs $311 / \mathrm{en} /$

3. Adami F, Vasconcelos FAG. Obesidade na infância e adolescência e mortalidade na idade adulta: uma revisão sistemática de estudos de coorte. Cad Saúde Pública 2008; 24(Supl. 4):558-568.

4. Lee YS. Consequences of childhood obesity. Ann Acad Med Singapore 2009; 38(1):75-81.

5. Leal VS, Lira PIC, Oliveira JS, Menezes RCE, Sequeira LAS, Arruda Neto MA, Andrade SLLS, Batista Filho M. Excesso de peso em crianças e adolescentes no Estado de Pernambuco, Brasil: prevalência e determinantes. Cad Saúde Pública 2012; 28(6):1175-1182.

6. Anjos LA, Castro IRR, Engstrom EM, Azevedo AMF. Crescimento e estado nutricional em amostra probabilística de escolares no Município do Rio de Janeiro, 1999. Cad Saúde Pública 2003; 19(1):171-179.

7. Costa RF, Cintra IP, Fisberg M. Prevalência de Sobrepeso e Obesidade em Escolares da Cidade de Santos, SP. Arq Bras Endocrinol Metabol 2006; 50(1):60-67.

8. Moreira MA, Cabral PC, Ferreira HS, Lira PIC. Excesso de peso e fatores associados em crianças da região nordeste do Brasil. J Pediatr 2012; 88(4):347-352.

9. Instituto Brasileiro de Geografia e Estatística (IBGE) Pesquisa de Orçamentos Familiares 2008-2009: Antropometria e Estado Nutricional de Crianças, adolescentes e adultos no Brasil. Rio de Janeiro: IBGE; 2010.

10. Bernardo CO, Vasconcelos FAG. Association of parents nutritional status, and sociodemographic and dietary factors with overweight/obesity in schoolchildren 7 to 14 years old. Cad Saúde Pública 2012; 28(2):291-304.

11. Uauy R, Diaz E. Consequences of food energy excess and positive energy balance. Public Health Nutr 2005; 8(7A):1077-1099.

12. Enes CC, Slater B. Obesidade na adolescência e seus principais fatores determinantes. Rev Bras Epidemiol 2010; 13(1):163-171.

\section{Colaboradores}

CE Rossi realizou as análises estatísticas e elaborou o manuscrito. LCF Costa colaborou com a realização das análises estatísticas e com a elaboração do manuscrito. MS Machado delineou o estudo e colaborou com a revisão do manuscrito. DF Andrade colaborou com as análises estatísticas e aprovou a versão final do artigo. FAG Vasconcelos colaborou com a revisão do manuscrito e aprovação da versão final.
13. Bray GA, Popkin BM. Dietary fat intake does affect obesity. Am J Clin Nutr 1998; 68(6):1157-1173.

14. Ramos M, Stein LM. Desenvolvimento do comportamento alimentar infantil. J Pediatr 2000; 64 (Supl. 3):229-237.

15. World Health Organization (WHO). Global strategy on diet, physical activity and health. Fifty-seventh World Health Assembly. Geneva: WHO; 2004.

16. Ippolito-Shepherd J, Cerqueira MT, Ortega DP. Iniciativa Regional Escuelas Promotoras de la Salud en las Américas. IUHPE - Promotion \& Education 2005; XII:220-229. [acessado 2008 Out 25]. Disponível em: http://www.bvsde.paho.org/bvsacd/cd57/EPS_IUHPE. pdf

17. Moura JBVS, Lourinho LA, Valdês MTM, Frota MA, Catrib AMF. Perspectiva da epistemologia histórica e a escola promotora de saúde. Hist Cienc Saúde Manguinhos 2007; 14(2):489-501.

18. American Dietetic Association (ADA). Position of the American Dietetic Association: local nutrition guidance for health children ages 2 to 11 years. J Am Diet Assoc 2008; 1(108):1038-1047.

19. Bell AC, Swinburn BA. What a key food groups to targed for preventing obesity and improving nutrition in schools? Eur J Clin Nutr 2004; 58(2):258-263.

20. Temple JL, Steyn NP, Myburgh NG, Nel JH. Overweight children find food more reinforcing and consume more energy than do nonoverweight children. J Am Coll Nutr 2006; 87(5):1121-1127.

21. Fox MK, Gordon A, Nogales R, Wilson A. Availability and consumption of competitive foods in US public schools. J Am Diet Assoc 2009; 109(Supl. 2):57-66.

22. Fox MK, Dodd AH, Wilson A, Gleason PM. Association between school food environment and practices and body mass index of US public school children. $J$ Am Diet Assoc 2009; 109(Supl. 2):108-117.

23. Kubik MY, Lytlle LA, Story M. School wide food practices are associated with Body Mass Index in middle school students. Arch Pediatrics Adolesc Med 2005; 159(12):1111-1114. 
24. Brasil. Ministério da Saúde (MS). Manual operacional para profissionais de saúde e educação: promoção da alimentação saudável nas escolas. Brasília: MS; 2008. (Série A. Normas e Manuais Técnicos).

25. Gabriel CG, Vasconcelos FAG, Andrade DF, Schmitz BAS. First Law regulating school canteens in Brazil: evaluation after seven years of implementation. ALAN 2009: 59(2):128-138.

26. Ricardo GD, Caldeira GV, Corso ACT. Prevalência de sobrepeso e obesidade e indicadores de adiposidade central em escolares de Santa Catarina, Brasil. Rev Bras Epidemiol 2009: 12(3):424-435.

27. Costa LCF, Vasconcelos FAG, Corso ACT. Fatores associados ao consumo adequado de frutas e hortaliças em escolares de Santa Catarina, Brasil. Cad Saude Publica 2012; 28(6):1133-1142.

28. Fonseca MJM, Faerstein E, Chor D, Lopes CS. Validade de peso e estatura informados e índice de massa corporal: estudo pró-saúde. Rev Saúde Pública 2004; 38(3):392-398.

29. World Health Organization (WHO). Physical status: the use and interpretation of anthropometry. Geneva: WHO; 1995.

30. Cole TJ, Bellizzi MC, Flegal KM, Dietz WH. Establishing a standard definition for child overweight and obesity worldwide: international survey. BMJ 2000; 320(7244):1240-1243.

31. Assis MAA, Bendet J, Kerpel R, Vasconcelos FAG, Di Pietro PF, Kupek E. Validação da Terceira versão do Questionário Alimentar do dia anterior (QUADA -3) para escolares de 6 a 11 anos. Cad Saúde Pública 2009; 25(8):1816-1826.

32. Hulley SB, Cummings SR, Browner WS, Grady DG, Newman TB. Designing Clinical Research. $4^{\text {th }}$ ed. Philadelphia: Wolters Kluwer Health, Lippincott Williams \& Wilkins; 2013.

33. Brasil. Lei ${ }^{\circ} 11.947$, de 16 de junho de 2009. Dispõe sobre o atendimento da alimentação escolar e do Programa Dinheiro Direto na Escola aos alunos da educação básica. Diário Oficial da União 2009; 17 jun.

34. Instituto Brasileiro de Geografia e Estatística (IBGE). Pesquisa Nacional de Saúde do Escolar (PeNSE) - 2012. Rio de Janeiro: IBGE; 2013.

35. Instituto Brasileiro de Geografia e Estatística (IBGE). Pesquisa Nacional de Saúde do Escolar (PeNSE) - 2015. Rio de Janeiro: IBGE; 2016.

36. Gabriel CG, Santos MV, Vasconcelos FAG. Avaliação de um programa para promoção de hábitos alimentares saudáveis em escolares de Florianópolis, Santa Catarina, Brasil. Rev Bras Saúde Matern Infant 2008; 8(3):299-308.

37. Kuntz MGF, Fiates GMR, Teixeira E. Healthy and tasty school snacks: suggestions from Brazilian children consumers. Int J Consumer Studies 2012; 36(1):38-43.

38. Togoo RA, Meer Z, Kandlaya R, Al-Shehri SMYTD, Al-Ghamdi HG. Availability of cariogenic foods in primary school canteens of Albha City, Saudi Arabia: A Cross-Sectional Study. World J Dent 2012; 3(3):239242 .
39. Brasil. Ministério da Saúde (MS), Ministério da Educação (MEC). Portaria Interministerial no 1.010 , de 8 de maio de 2006. Institui as diretrizes para a Promoção da Alimentação Saudável nas Escolas de educação infantil, fundamental e nível médio das redes públicas e privadas, em âmbito nacional. Diário Oficial da União 2006; 9 maio.

40. Santa Catarina. Lei n. 12.061, de 18 de dezembro de 2001. Dispõe sobre critérios de concessão de serviços de lanches e bebidas nas unidades educacionais, localizadas no Estado de Santa Catarina. Diário Oficial do Estado de Santa Catarina 2001; $20 \mathrm{dez}$.

41. Brasil. Resolução CD/FNDE n' 26, de 17 de junho de 2013. Dispõe sobre o atendimento da alimentação escolar aos alunos da educação básica no âmbito do programa Nacional de Alimentação Escolar - PNAE. Diário Oficial da União 2013; 18 jun.

42. Brasil. Resolução CD/FNDE n 32 , de 10 de agosto de 2006. Estabelece as normas para a execução do Programa Nacional de Alimentação Escolar - PNAE. Diário Oficial da União 2006; 11 ago.

43. Condon EM, Crepinsek MK, Fox MK. School Meals: Types of Foods Offered to and Consumed by Children at Lunch and Breakfast. J Am Diet Assoc 2009; 109(Supl. 2):67-78.

44. Nicklas TA, Morales M, Linares A, Yang SJ, Baranowski T, Moor C, Berenson G. Children's meal patterns have changed over a 21-year period: The Bogalusa Heart Study. J Am Diet Assoc 2004; 104(5):753-761.

45. Moraes AS, Rosas JB, Mondini L, Freitas ICM. Prevalência de sobrepeso e obesidade e fatores associados em escolares de área urbana de Chilpancingo, Guerrero, México, 2004. Cad Saúde Pública 2006; 22(6):12891301.

46. Jehn ML, Gittelsohn J, Treuth MS, Caballero, B. Prevalence of overweight among Baltimore city schoolchildren and its associations with nutrition and physical activity. Obes 2006; 14(6):989-993.

47. Utter J, Schaaf D, Mhurchu CN, Scragg R. Food choices among students using the school food service in New Zealand. NZMJ 2007; 1248(120):11.

48. Gleason PM, Dodd AH. School breakfast program but not school lunch program participation is associated with lower body mass index. J Am Diet Assoc 2009; 109(Supl. 2):118-128.

Artigo apresentado em 24/10/2016

Aprovado em 19/04/2017

Versão final apresentada em 21/04/2017 